\title{
Racial Disparities and Cardiovascular Disease: One Size Fits All Approach?
}

\author{
Santoshi Ashwitha ${ }^{1}$, Alexandra Wee ${ }^{1}$, Manasi Shirke ${ }^{1}$, and Amer Harky² \\ ${ }^{1}$ Queen's University Belfast, School of Medicine, , UK \\ ${ }^{2}$ Liverpool Heart and Chest Hospital NHS Foundation Trust
}

September 2, 2020

\begin{abstract}
Background: Despite recent advancements in prevention, treatment, and management options, cardiovascular diseases contribute to one of the leading causes of morbidity and mortality. Several studies highlight the compelling evidence for the existence of healthcare inequities and disparities in the treatment and management control of cardiovascular diseases. Aims: To explore the role of racial disparities in the treatment of various cardiovascular diseases, highlighting the role of socioeconomic and cultural factors, and ultimately postulate solutions to eliminate the disparities. Methods: A comprehensive review of literature was conducted using appropriate keywords on search engines of SCOPUS, Wiley, PubMed, and SAGE Journals. Conclusion: By continued research to eliminate healthcare inequalities, there exists a potential to improve health-related outcomes in minority populations.
\end{abstract}

\section{Abstract}

Background: Despite recent advancements in prevention, treatment, and management options, cardiovascular diseases contribute to one of the leading causes of morbidity and mortality. Several studies highlight the compelling evidence for the existence of healthcare inequities and disparities in the treatment and management control of cardiovascular diseases.

Aims: To explore the role of racial disparities in the treatment of various cardiovascular diseases, highlighting the role of socioeconomic and cultural factors, and ultimately postulate solutions to eliminate the disparities.

Methods: A comprehensive review of literature was conducted using appropriate keywords on search engines of SCOPUS, Wiley, PubMed, and SAGE Journals.

Conclusion: By continued research to eliminate healthcare inequalities, there exists a potential to improve health-related outcomes in minority populations.

\section{Introduction}

Cardiovascular diseases (CVD) contribute significantly to the rising cases of morbidity and mortality in Western countries while being a major health threat in the developing world. CVD encompasses diagnoses including, but not limited to, Coronary Heart Disease (CHD), strokes, aortic disease, and peripheral arterial disease (PAD). ${ }^{1}$ There exists a positive correlation between risk factors, such as hypertension, obesity, smoking, hypercholesteremia, and CVD. According to the World Health Organisation (WHO), there have been approximately 17.9 million annual deaths from CVD, which represents $31 \%$ of deaths worldwide. ${ }^{2,3}$

Despite remarkable advancements in risk factor identification, and the widespread implementation of evidence-based strategies for CVD management, racial health disparities persist, which play an indisputable role in predisposing minorities to CVD. ${ }^{1}$ For instance, with data from the United States (US) and the United 
Kingdom (UK), CVD accounts for more than one-third of the differences in mortality rates in ethnic minorities such as Black people compared to White. ${ }^{4,5}$ The Institute of Medicine (IOM) report, Unequal treatment: confronting racial and ethnic disparities in health care, defines health disparities as "racial or ethnic differences in the quality of healthcare that are not due to access-related factors or clinical needs, preferences, and appropriateness of intervention." This definition recognises the role of synergistic factors, such as limited health literacy and healthcare access, inadequate cultural competency and implicit negative biases amongst physicians as mediators of racial/ethnic disparities. ${ }^{6}$

The purpose of this review is to focus on the drawbacks of past CVD research, explore the role of racial disparities in the treatment of Hypertension, Coronary Artery Disease, Arrhythmias, Heart Failure, and , highlighting the role of socioeconomic and cultural factors in CVD, and provide future directions to eliminate these disparities in the global population.

\section{Gaps in Existing Research}

Numerous epidemiological studies have documented African-American disparities in CVD, focusing on the interplay of patient-level, healthcare, and environmental risk factors. Alongside the study of these vital factors, there has to be equal emphasis on the primordial factors (pathophysiological mechanisms) which underlie these racial disparities. Comprehensive understanding of the in-depth mechanisms can help devise strategies to improve management and treatment of CVD, promoting equity. ${ }^{7}$

The ultimate goal of clinical research is to devise strategies that aim to prevent and treat disease, where the study population is representative of the affected population. However, a majority of the CVD populationbased studies (USA) have been largely limited to White-American and African-American cohorts. Underrepresentation in medical research has far-reaching implications, that significantly contribute to healthcare disparities. As the nation's population begins to grow, with an increasingly diverse and multicultural patient base, clinical studies have to incorporate other racial sub-groups, such as Asian-American cohorts, to better reflect CVD trends in the general population, which will enable generalisability of results to minority subgroups. ${ }^{8}$

A prospective, observational study assessed those at risk for or with atherothrombotic disease (CAD, PAD, and CVD) from seven racial/ethnic sub-groups A 2-year follow up highlighted that Blacks had the highest cardiovascular mortality, and Asians had the lowest cardiovascular mortality. The study, however, does not define the underlying aetiologies (social, environmental, cultural factors) amongst the ethnic sub-groups, and their contribution to the cardiovascular outcomes, which could be a potential prospect for future research. ${ }^{9}$

Furthermore, a large number of CVD cohort studies comprised of small sample sizes and were limited to singular healthcare organisations, which affected the generalisability of results. There exists wide variation in components of the intervention, with varying study designs, contributing to a lack of overall crossstandardisation, impacting the interpretability of results, and increased the difficulty to draw broad conclusions across the studies. Considering the retrospective nature of majority CVD studies, there also arose elements of recall, publication, and acquiescence biases ${ }^{10}$

Considering the important shortcomings of past research, a great deal of work to reduce healthcare disparities to bridge the translation and fundamental knowledge gaps is essential.

\section{Disparities in treatments:}

\section{Hypertension}

The term "hypertension" refers to arterial hypertension characterised by a systolic blood pressure (SBP) exceeding $140 \mathrm{mHg}$, and diastolic blood pressure (DBP) exceeding 90mmHg. "Treatment" shall refer to generally adopted prescriptions of antihypertensive drugs such as oral anticoagulants, diuretics, and digoxin and exclude lifestyle or dietary measures. ${ }^{11}$

Several US and UK studies report a higher prevalence and severity of hypertension in ethnic minorities such as South Asian, Caribbean, and West African populations compared to White subjects, which is in line with 
recent JBS guidelines. ${ }^{12}$ Studies used for this review have been summarised in Table 1.

UK and US clinical guidelines indicate that patients of African/Caribbean origin should be offered calcium channel blockers as first-line treatment, while patients aged below 55, not of African-Caribbean origin, should be offered an angiotensin-converting enzyme inhibitor (ACEI) or an angiotensin receptor antagonist (ARB). ${ }^{18}$ European hypertension treatment guidelines have only recently adopted similar specificity in drug treatments, however, neglect other ethnic minorities (e.g., South Asians, Chinese), and conclude that no evidence exists to prove that response to treatment differs from the general European population. ${ }^{11}$

The specialised approach to treatment in Black patients has been attributed to a multitude of physiological attributes such as higher circulatory levels of endothelin-1 and weaker vasodilation in response to nitric oxide compared to White patients. ${ }^{19}$ A comparative analysis of data from six randomised controlled trials on the effect of endothelin receptor antagonists (ERAs) on treatment outcomes measured by the distance patients could walk in six minutes found that White patients had an increased walk distance compared to Black patients. This study hypothesised these results to be due to an insufficient ERA dosage in Black patients $^{20}$ to compensate for the increased levels of endothelin-1. Other physiological attributes affecting response to treatment in other ethnicities include variations in the phenotypic and genotypic expression of cytochrome P450 enzymes. ${ }^{21}$

The efficacy of pharmacological treatments has been minimally investigated in minority ethnicities. Per clinical guidelines, ACEIs have been proven to be less effective in Black patients due to increased risk of morbidities such as stroke and heart failure, while those prescribed thiazide-like diuretics achieved better falls in blood pressure. ${ }^{22}$ Brewster et al. analysed pooled data on the ability of antihypertensive drug therapy in lowering blood pressure and did not find any evidence to suggest a differing efficacy of hypertension drugs in South Asians. ${ }^{23}$ A lack of resources in that area renders the data inconclusive. More research needs to be conducted on not only drug efficacy in the South Asian population but also rates of morbidity and mortality following drug therapy.

Most studies recognise that ethnic minorities do not meet treatment goals or receive treatment despite eligibility for hypertension medication. A major challenge in achieving control is thought to be medical compliance. $^{24}$ Various sources have uncovered lower rates of adherence in Black patients in comparison to White patients ${ }^{25}, 26$, a trend synonymous with several other ethnic minorities such as Chinese and South Asians. $^{27}$

\section{Coronary Artery Disease}

Coronary Artery Disease (CAD/CHD) is characterised by the narrowing of the coronary arteries, reducing blood flow and leading to angina, while complete blockage may induce a heart attack. Treatment for CAD currently comprises medication, surgical procedures, or both depending on the severity and extent of the disease. $^{28}$ Studies used for the review have been summarised in Table 2.

A higher incidence of CAD has been reported in South Asian patients than other ethnic minorities in the UK, which remains in line with Joint British Societies (JBS) guidelines of date. ${ }^{12}$ These guidelines also detail the lower risk of the African-Caribbean population of CAD by comparison.

Although treatment guidelines are universal across all ethnicities, the age whereby the patient would require treatment and efficacy of the treatment is variable. Iantorno et al., in his retrospective analysis, compared Black women who had undergone percutaneous coronary intervention (PCI) in response to conditions caused by CAD (e.g., stable angina and acute coronary syndrome) with White women of the same circumstance. Results indicated that Black women were younger and had more risk factors than Whites and were more likely to present with acute coronary syndrome. ${ }^{34}$ These results reiterated the findings of a different study comparing Black and White patients undergoing isolated CABG, which indicated that black patients were younger, with more comorbidities, and presented more severely than their White counterparts. ${ }^{35}$ Population studies have also proven that Hispanic patients undergoing CABG are generally younger than their White counterparts. ${ }^{36}$ 
Some studies also report disparities experienced by ethnic minorities in terms of quality and accessibility to treatment. One study pointed out the lower likelihood of Black patients with acute MI being admitted to a facility capable of revascularisation ${ }^{37}$, while another found Black patients at the receiving end of longer revascularisation times, notably more so if they were also male ${ }^{38}$. In addition to poor accessibility, minority ethnic groups have had similar access to healthcare but remained less likely to undergo revascularisation or cardiac procedures. ${ }^{39}$

A US study comparing postoperative complications 30 days following the procedure in Black, White and Asian patients who had undergone CABG found that Black and Asian patients had higher rates of postoperative pulmonary morbidity and mortality. The same ethnic minorities also had longer hospital stays post-op. ${ }^{40}$

A deficiency in the literature on other ethnic minorities has proven to be limiting in terms of fully evaluating treatment responses and where literature has been published, it has been overwhelmingly focused on Asian migrant populations in the Western world as opposed to native Asian populations. ${ }^{40,41}$ It has been noted in various papers that additional research on racial disparities in treatment is detrimental in tailoring healthcare better suited to each patient and improving treatment outcomes in populations that only continue to become increasingly diverse. ${ }^{42}$ This is a theme Gijsberts et al. have aimed to cater to in their multivariable Cox regression analysis comparing mortality in patients undergoing coronary angiography, revascularisation or CAD in the Netherlands and patients in Singapore, consisting of three large Southeast Asian ethnic subgroups, Malays (highest mortality rate), Chinese and Indians. ${ }^{43}$

\section{Arrhythmias}

Arrhythmias are abnormalities of the electrical properties of the heart which causes a change in the rhythm and/or heartbeat rate. Arrhythmias can be categorised on the basis of site and cardiac frequency.Treatment for arrhythmias include anti-arrhythmic medication, catheter ablation, cardioversion, implantable pacemakers and cardioverter-defibrillators (ICD). ${ }^{44}$ This section will discuss treatments for atrial fibrillation (AF) given its overall prevalence in developed countries and published literature. Table 3 summarises the studies used for the purpose of this review.

The incidence of AF does not appear to differ between ethnicities, some studies even noting a higher incidence of $\mathrm{AF}$ in White patients over patients from ethnic minorities. ${ }^{50,51}$ Conversely however, Black patients have a higher prevalence of risk factors associated with AF compared to their White counterparts, a phenomenon referred to as the 'racial paradox'. ${ }^{52,53}$ The mechanism is poorly understood and requires further research to ascertain causes and effects.

The main barriers to treatment for minority ethnics exist in the racial disparities surrounding patient awareness and treatment efficacy. Awareness of having AF and its clinical implications is significantly lower in Black patients compared to Whites. Furthermore, studies have noted that Black patients were about one fourth as likely to be prescribed warfarin than Whites. ${ }^{46}$ This likely due to the lower efficacy of warfarin treatment on AF in Black patients, with studies noting the increased dosage requirement and shorter time in therapeutic range (TTR) of internalised normal ratio (INR). ${ }^{49,54}$

Inconsistent results have been yielded from the various studies that have been performed evaluating the reduced prescription of non-vitamin $\mathrm{K}$ oral anticoagulants (NOACs) in minority ethnics. ${ }^{50,53} \mathrm{~A} 2018$ study comparing the rates of prescription of OACs among Black, White, and Hispanic AF patients calculated a $27 \%$ decrease in the likelihood of receiving NOACs in Black patients but deemed that figure insignificant after accounting for socioeconomic factors. ${ }^{55}$ A recent 2020 study, however, found that Black, Asian, and other minority ethnics were in fact, significantly less likely to receive any OACs, the difference continuing to be significant even after accounting for socioeconomic factors. ${ }^{56}$ The efficacy and required doses of OACs have not been performed in populations with adequate representation of ethnic minorities, and therefore cannot be commented on at this time. ${ }^{53,56}$

Several studies have also noted that non-White populations are less likely to be treated with rhythm control 
modalities and are instead treated with a rate control modality. Following rhythm control modalities, White patients were more likely to undergo cardioversion, be prescribed antiarrhythmic medication, and undergo interventional AF procedures, such as catheter ablation and surgery than Black and Hispanic patients. They also had higher implantation rates of cardioverter defibrillators and permanent pacemakers. ${ }^{48,49}$

Some studies attribute these disparities to the poorer access to healthcare experienced by minority ethnics, which have been theorised to be a consequence of lower literacy, cultural norms, and language barriers. This is thought to lead to a lack of recruitment of ethnic minorities in clinical trials. ${ }^{57}$ Further research seems necessary in discerning the causation of these differences.

\section{Heart Failure}

Heart failure (HF) is a circulatory condition caused by structural and functional changes that adversely affect the pumping (systole) and filling (diastole) of the heart leading to elevated intracardiac pressures or reduced resting cardiac output resulting in a myriad of symptoms. HF can be acute (reduced left ventricular ejection filling) or chronic (preserved left ventricular ejection fraction). Treatment for heart failure currently range from medications to device implantations(pacemakers, ICDs) and surgical procedures. ${ }^{58}$

Various sources comment on the high prevalence and susceptibility of HF in patients of African descent. ${ }^{58}$ Black and Hispanic patients in the US and South Asians in the UKhave also been found to develop HF at a younger age. Black patients generally have more adverse treatment outcomes. More recent studies examining HF prevalence in UK ethnic minorities have not been performed. Overall, more research needs to be done to identify the determinants of outcome differences across ethnicities. A summary of studies used for the purpose of this review have been detailed in Table 4.

The efficacy of pharmacological treatment across ethnicities have yet to be explored adequately especially non-Black ethnic minorities. HF drugs commonly used unless contraindicated include ACEIs, ARBs and diuretics. Many sources have reported reduced efficacy of ACEIs and spironolactone in Blacks, while conflicting results have been obtained from studies involving beta blockers. ${ }^{67,68}$ Shekelle et al.'s meta-analysis of four randomised controlled trials (RCTs) of beta blockers would estimate the relative risk (RR) of mortality to be 0.97 in Blacks and 0.69 in Whites. However, upon removal of data from one of the four RCTs, the RR of black patients reduced to 0.67 . This was hypothesised to be due to the small sample size of black patients in the analysis, a weakness experienced by many other studies. ${ }^{69}$

Of note, the addition of combinedhydralazine and isosorbide dinitrate is recommended for use in Black patientsby the European Society of Cardiology (ESC) following evidence of reduced morbidity and mortality rates in black HF patients. ${ }^{70}$ Given the hypothesis that reduced efficacy of ACEIs in Black patients is due to reduced bioactivity of nitric oxide (NO), these results are thought to emphasise using $\mathrm{NO}$ enhancing therapies in Black patients. ${ }^{19,71}$ The increase in efficacy has only been clearly examined and proven in Black patients, due to scarcity of literature on other ethnic minorities. ${ }^{70}$

Medical compliance to HF drugs is also a factor in predicting outcomes in ethnic minorities. In the US, an observational study comparing medical adherence across ethnicities found that Native Americans, Black, Hispanic and Asian patients had lower rates of adherence than their White counterparts despite drug coverage by Medicare which is suggestive of other factors such as health beliefs surrounding adherence and eventual outcomes of treatment. ${ }^{72}$

\section{Role of socioeconomic \& cultural factors}

Various studies have utilised a variety of approaches to conceptualise and measure the inverse relationship between SES and the risk of CVD, in terms of education, employment status, occupation, annual income and social class.

Intensive studies concerning pathophysiology of atherosclerosis have shed light on the environmental contribution to atherosclerotic disease and CVD alongside non-modifiable risk factors, such as age and genetics. Variation in the geographical location, and diversity of cultural and social practices alter CVD risk. $\mathrm{Cu}-$ 
mulative data from the Nurse's Health Study ${ }^{73}$ and the Health Professionals follow-up study suggested that $82 \%$ and $62 \%$ of coronary events, respectively could have been avoided if the study participants adhered to a healthy, low-risk lifestyle. However, obscurity of the cumulative impact of various environmental factors and mechanisms influencing CVD limits the validity of these studies. ${ }^{74}$

A systematic review conducted to study disparities in CVD care highlighted gaps in well-established markers, such as SES which is an important social determinant of health. ${ }^{5}$ Recent studies have established good evidence regarding modifiable factors, such as SES and cultural factors which act as stronger determinants of health-related outcomes when compared to race. Likewise, the studies emphasised on the diminishing effect of disparate care when factors, such as SES are controlled for, which eventually leads to the influence of race being zeroed down. ${ }^{75}$ The reviews have identified an inverse relationship between education and CVD, with lower educational attainment being associated with higher incidence of hypertension, physical inactivity, sedentary lifestyle which contribute as risk factors to CVD.

A large-scale cohort study, the Prospective Urban Rural Epidemiologic (PURE) study recruited adults aged between 35 and 70 years from low-income, middle-income and high-income countries to explore the influence of economic levels on the CVD mortality. The results suggested the presence of an inverse relationship between SES and CVD mortality, with the gradient being steepest for the low-income countries. The study also concluded the greater influence of the educational factor than the household income on CVD incidence and death rate, with associations of poorer access to healthcare systems in low-income and middle-income countries. ${ }^{76}$

Clinical patient data from the Hypertension Detection and Follow-up program (HDFP) examined the association between SES, measured in terms of education and the prevalence of hypertension in Black and White racial groups. As anticipated, education was inversely associated with incidence on hypertension in both the racial groups, irrespective of sex. The relationship was more pronounced, however, in the younger Black patient sub-group compared to the Whites. Nevertheless, SES reflected in terms of education alone couldn't completely explain the association with CVD, as despite equal educational attainment between both patient sub-groups, greater cases of hypertension were reported in the Blacks. These fluctuations could be explained in terms of other contributing environmental factors and genetic factors, such as Vitamin D levels. ${ }^{74}$

A community-based cross-sectional study of 347 adults aged 18 to 17 years old in Nepal concluded an increased risk of CVD in individuals without any educational background. ${ }^{77}$ Moreover, a prospective UK SABRE analysis of a cohort of 1090 Europeans and 1006 South Asians studied the association of four healthy behaviours (high fibre diet, non-smoker, moderate alcohol consumption, and physically active) and CVD outcomes. Over a 21-year follow-up, lower adherence to the four healthy behaviours led to a 2 to 3-fold increased incidence CHD risk in both ethnic groups.$^{78}$

A conclusive overview of the studies emphasizes on the significant contribution of SES to the aetiology, development of CVD and subsequent mortality and morbidity.

\section{Future Perspectives}

The identification of behavioural determinants of CVD will aid in the designing of future programs and applications to address these health determinants and reduce risky behaviors. There also exists a need for research aimed at understanding the psychological impact of racial and/or ethnic disparities on CVD health related outcomes. Research in this field could potentially benefit future public health and clinical practices. ${ }^{79}$

African Americans, and Hispanic Americans account for $13 \%$ and $16 \%$ of the US population, however, the racial sub-groups only contribute to $9 \%$ of the physician populace. Due to the underrepresentation in the workforce, African Americans and Hispanic Americans are more likely to be treated by physicians from different ethnic/racial background compared to Whites. ${ }^{8}$ Evidence from a comprehensive review supports patient-provider race-concordance and its association with positive health outcomes in minorities. ${ }^{80}$ Similarly, medical education programs can greatly benefit cultural competence of physicians, helping them acquire skills to interact with the marginalised patient groups, subsequently contributing to the elimination of health 
disparities. ${ }^{79}$

\section{Conclusion}

There exists compelling evidence of the pervasive nature on disparities in cardiovascular health, which are prevalent in population sub-groups defined by race, gender, ethnicity, socioeconomic status (SES), subsequently contributing to the adverse impact on quality of life (QOL) and medical outcomes. To eliminate these CVD disparities in minority populations, greater efforts focused on guideline adherence, risk factor control and management are required.

\section{Human Studies: No ethical approval required as no patient information was shared}

\section{References}

[1] Muncan B. Cardiovascular disease in racial/ethnic minority populations: illness burden and overview of community-based interventions. Public Health Rev [Internet]. 2018;39(1). Available from: http://dx.doi.org/10.1186/s40985-018-0109-4

[2] Cardiovascular disease [Internet]. Nhs.uk. Available from: https://www.nhs.uk/conditions/cardiovascular-disease/. Accessed August 23, 2020.

[3] Cardiovascular diseases (CVDs) [Internet]. Who.int. Available from: https://www.who.int/en/newsroom/fact-sheets/detail/cardiovascular-diseases-(cvds). Accessed August 23, 2020.

[4] Francis DK, On behalf of the U.S. Caribbean Alliance for Health Disparities Research Group (USCAHDR), Bennett NR, Ferguson TS, Hennis AJM, Wilks RJ, et al. Disparities in cardiovascular disease among Caribbean populations: a systematic literature review. BMC Public Health [Internet]. 2015;15(1). Available from: http://dx.doi.org/10.1186/s12889-015-2166-7

[5] Cooper R, Cutler J, Desvigne-Nickens P, Fortmann SP, Friedman L, Havlik R, et al. Trends and disparities in coronary heart disease, stroke, and other cardiovascular diseases in the United States: Findings of the national conference on cardiovascular disease prevention. Circulation. 2000;102(25):3137-47.

[6] McGuire TG, Alegria M, Cook BL, Wells KB, Zaslavsky AM. Implementing the Institute of Medicine definition of disparities: an application to mental health care. Health Serv Res. 2006;41(5):1979-2005.

[7] Hackler E III, Lew J, Gore MO, Ayers CR, Atzler D, Khera A, et al. Racial differences in cardiovascular biomarkers in the general population. J Am Heart Assoc [Internet]. 2019;8(18). Available from: http://dx.doi.org/10.1161/jaha.119.012729

[8] Youmans QR, Hastings-Spaine L, Princewill O, Shobayo T, Okwuosa IS. Disparities in cardiovascular care: Past, present, and solutions. Cleve Clin J Med. 2019;86(9):621-32.

[9] Meadows TA, Bhatt DL, Cannon CP, Gersh BJ, Röther J, Goto S, et al. Ethnic differences in cardiovascular risks and mortality in atherothrombotic disease: insights from the Reduction of Atherothrombosis for Continued Health (REACH) registry. Mayo Clin Proc. 2011;86(10):960-7.

[10] Davis AM, Vinci LM, Okwuosa TM, Chase AR, Huang ES. Cardiovascular health disparities. Med Care Res Rev. 2007a;64(5_suppl):29S-100S.

[11] 2018 Practice Guidelines for the management of arterial hypertension of the European Society of Hypertension and the European Society of Cardiology. Journal of Hypertension. 2019;37(2):456.

[12] Joint British Societies' consensus recommendations for the prevention of cardiovascular disease (JBS3). Heart. 2014;100(Suppl 2):ii1-ii67.

[13] Cappuccio F, Cook D, Atkinson R, Strazzullo P. Prevalence, detection, and management of cardiovascular risk factors in different ethnic groups in south London. Heart. 1997;78(6):555-563. 
[14] Dorans K, Mills K, Liu Y, He J. Trends in Prevalence and Control of Hypertension According to the 2017 American College of Cardiology/American Heart Association (ACC/AHA) Guideline. Journal of the American Heart Association. 2018;7(11).

[15] George, MG, Schieb, LJ, Ayala, C Pulmonary hypertension surveillance: United States, 2001 to 2010. Chest 2014; 146(2): 476-495.

[16] Kishi, S, Reis, JP, Venkatesh, BA Race-ethnic and sex differences in left ventricular structure and function: the Coronary Artery Risk Development in Young Adults (CARDIA) Study. J Am Heart Assoc 2015; 4(3): e001264[17] Yang BQ, Assad TR, O’Leary JM, Xu M, Halliday SJ, D'Amico RW, et al. Racial differences in patients referred for right heart catheterization and risk of pulmonary hypertension. Pulm Circ. 2018;8(2):2045894018764273.

[18] Elliott W. NICE clinical guideline 127: Hypertension: Clinical management of primary hypertension in adults. Yearbook of Cardiology. 2012;2012:1-3.

[19] Campia U, Choucair W, Bryant M, Waclawiw M, Cardillo C, Panza J. Reduced endothelium-dependent and -independent dilation of conductance arteries in African Americans. Journal of the American College of Cardiology. 2002;40(4):754-760.

[20] Gabler N, French B, Strom B, Liu Z, Palevsky H, Taichman D et al. Race and Sex Differences in Response to Endothelin Receptor Antagonists for Pulmonary Arterial Hypertension. Chest. 2012;141(1):20-26.

[21] Myrand S, Sekiguchi K, Man M, Lin X, Tzeng R, Teng C et al. Pharmacokinetics/Genotype Associations for Major Cytochrome P450 Enzymes in Native and First- and Third-generation Japanese Populations: Comparison With Korean, Chinese, and Caucasian Populations. Clinical Pharmacology \& Therapeutics. 2008;84(3):347-361.

[22] Johnson J, Boerwinkle E, Zineh I, Chapman A, Bailey K, Cooper-DeHoff R et al. Pharmacogenomics of antihypertensive drugs: Rationale and design of the Pharmacogenomic Evaluation of Antihypertensive Responses (PEAR) study. American Heart Journal. 2009;157(3):442-449.

[23] Brewster L, van Montfrans G, Oehlers G, Seedat Y. Systematic review: antihypertensive drug therapy in patients of African and South Asian ethnicity. Internal and Emergency Medicine. 2016;11(3):355-374.

[24] Ritchey M, Chang A, Powers C, Loustalot F, Schieb L, Ketcham M et al. Vital Signs: Disparities in Antihypertensive Medication Nonadherence Among Medicare Part D Beneficiaries — United States, 2014. MMWR Morbidity and Mortality Weekly Report. 2016;65(36):967-976.

[25] Lauffenburger J, Robinson J, Oramasionwu C, Fang G. Racial/Ethnic and Gender Gaps in the Use of and Adherence to Evidence-Based Preventive Therapies Among Elderly Medicare Part D Beneficiaries After Acute Myocardial Infarction. Circulation. 2014;129(7):754-763.

[26] Zhang Y, Baik S. Race/Ethnicity, Disability, and Medication Adherence Among Medicare Beneficiaries with Heart Failure. Journal of General Internal Medicine. 2013;29(4):602-607.

[27] Liu Q, Quan H, Chen G, Qian H, Khan N. Antihypertensive Medication Adherence and Mortality According to Ethnicity: A Cohort Study. Canadian Journal of Cardiology. 2014;30(8):925-931.

[28] Kandaswamy E, Zuo L. Recent Advances in Treatment of Coronary Artery Disease: Role of Science and Technology. International Journal of Molecular Sciences. 2018;19(2):424.

[29] Zaman M, Philipson P, Chen R, Farag A, Shipley M, Marmot M et al. South Asians and coronary disease: is there discordance between effects on incidence and prognosis?. Heart. 2013;99(10):729-736.

[30] Tillin T, Hughes A, Mayet J, Whincup P, Sattar N, Forouhi N et al. The Relationship Between Metabolic Risk Factors and Incident Cardiovascular Disease in Europeans, South Asians, and African Caribbeans. Journal of the American College of Cardiology. 2013;61(17):1777-1786. 
[31] Jose P, Frank A, Kapphahn K, Goldstein B, Eggleston K, Hastings K et al. Cardiovascular Disease Mortality in Asian Americans. Journal of the American College of Cardiology. 2014;64(23):2486-2494.

[32] King K, Khan N, Quan H. Ethnic Variation in Acute Myocardial Infarction Presentation and Access to Care. The American Journal of Cardiology. 2009;103(10):1368-1373.

[33] Zaman M, Crook A, Junghans C, Fitzpatrick N, Feder G, Timmis A et al. Ethnic differences in longterm improvement of angina following revascularization or medical management: a comparison between south Asians and white Europeans. Journal of Public Health. 2008;31(1):168-174.

[34] Iantorno M, Rogers T, Torguson R, Kolm P, Gajanana D, Khalid N et al. Racial Disparities in Clinical Characteristics and Outcomes of Women Undergoing Percutaneous Coronary Intervention. Cardiovascular Revascularization Medicine. 2019;20(12):1039-1042.

[35] Mehta R, Shahian D, Sheng S, O'Brien S, Edwards F, Jacobs J et al. Association of Hospital and Physician Characteristics and Care Processes With Racial Disparities in Procedural Outcomes Among Contemporary Patients Undergoing Coronary Artery Bypass Grafting Surgery. Circulation. 2016;133(2):124-130.

[36] van Ryn M, Burgess D, Malat J, Griffin J. Physicians' perceptions of patients' social and behavioural characteristics and race disparities in treatment recommendations for men with coronary artery disease. $\mathrm{Am}$ J Public Health . 2006;96(2):351-357.

[37] Mody P, Gupta A, Bikdeli B, Lampropulos J, Dharmarajan K. Most Important Articles on Cardiovascular Disease Among Racial and Ethnic Minorities. Circulation: Cardiovascular Quality and Outcomes. $2012 ; 5(4)$.

[38] Cavender M, Rassi A, Fonarow G, Cannon C, Peacock W, Laskey W et al. Relationship of Race/Ethnicity With Door-to-Balloon Time and Mortality in Patients Undergoing Primary Percutaneous Coronary Intervention for ST-Elevation Myocardial Infarction: Findings From Get With the Guidelines-Coronary Artery Disease. Clinical Cardiology. 2013;36(12):749-756.

[39] Albert MA, Ayanian JZ, Silbaugh TS, et al. Early results of Massachusetts healthcare reform on racial, ethnic, and socioeconomic disparities in cardiovascular care. Circulation 2014; 129(24):2528-2538.

[40] Burton B, Munir N, Labastide A, Sanchez R, Gabriel R. An Update on Racial Disparities With 30-Day Outcomes After Coronary Artery Bypass Graft Under the Affordable Care Act. Journal of Cardiothoracic and Vascular Anesthesia. 2019;33(7):1890-1898.

[41] Leigh J, Alvarez M, Rodriguez C. Ethnic Minorities and Coronary Heart Disease: an Update and Future Directions. Current Atherosclerosis Reports. 2016;18(2).

[42] Leigh J, Alvarez M, Rodriguez C. Ethnic Minorities and Coronary Heart Disease: an Update and Future Directions. Current Atherosclerosis Reports. 2016;18(2).

[43] Gijsberts C, Seneviratna A, de Carvalho L, den Ruijter H, Vidanapthirana P, Sorokin V et al. Ethnicity Modifies Associations between Cardiovascular Risk Factors and Disease Severity in Parallel Dutch and Singapore Coronary Cohorts. PLOS ONE. 2015;10(7):e0132278.

[44] Colilla S, Crow A, Petkun W, Singer D, Simon T, Liu X. Estimates of Current and Future Incidence and Prevalence of Atrial Fibrillation in the U.S. Adult Population. The American Journal of Cardiology. 2013;112(8):1142-1147.

[45] Shih T, Ledezma K, McCauley M, Rehman J, Galanter W, Darbar D. Impact of traditional risk factors for the outcomes of atrial fibrillation across race and ethnicity and sex groups. IJC Heart \& Vasculature. 2020;28:100538.

[46] Meschia J, Merrill P, Soliman E, Howard V, Barrett K, Zakai N et al. Racial Disparities in Awareness and Treatment of Atrial Fibrillation. Stroke. 2010;41(4):581-587. 
[47] Golwala H, Jackson L, Simon D, Fonarow G, Chang P, Gersh B et al. Racial/Ethnic Differences in Atrial Fibrillation Symptoms, Treatment Patterns and Outcomes: Insights from Outcomes Registry for Better Informed Treatment for Atrial Fibrillation (ORBIT-AF) Registry. Journal of the American College of Cardiology. 2015;65(10):A462.

[48] Mistry A, Vali Z, Sidhu B, Budgeon C, Yuyun M, Pooranachandran V et al. Disparity in implantable cardioverter defibrillator therapy among minority South Asians in the United Kingdom. Heart. 2020;106(9):671676.

[49] Yuyun M, Squire I, Ng G, Samani N. Evidence for reduced susceptibility to cardiac bradycardias in South Asians compared with Caucasians. Heart. 2018;104(16):1350-1355.

[50] Ugowe F, Jackson L, Thomas K. Racial and ethnic differences in the prevalence, management, and outcomes in patients with atrial fibrillation: A systematic review. Heart Rhythm. 2018;15(9):1337-1345.

[51] Khurshid S, Choi S, Weng L, Wang E, Trinquart L, Benjamin E et al. Frequency of Cardiac Rhythm Abnormalities in a Half Million Adults. Circulation: Arrhythmia and Electrophysiology. 2018;11(7)

[52] Stamos T, Darbar D. The "Double" Paradox of Atrial Fibrillation in Black Individuals. JAMA Cardiology. 2016;1(4):377.

[53] Amponsah M, Benjamin E, Magnani J. Atrial Fibrillation and Race - A Contemporary Review. Current Cardiovascular Risk Reports. 2013;7(5):336-345.

[54] Shen A, Chen W, Yao J, Brar S, Wang X, Go A. Effect of Race/Ethnicity on the Efficacy of Warfarin. CNS Drugs. 2008;22(10):815-825.

[55] Essien U, Holmes D, Jackson L, Fonarow G, Mahaffey K, Reiffel J et al. Association of Race/Ethnicity With Oral Anticoagulant Use in Patients With Atrial Fibrillation. JAMA Cardiology. 2018;3(12):1174.

[56] Tedla Y, Schwartz S, Silberman P, Greenland P, Passman R. Racial Disparity in the Prescription of Anticoagulants and Risk of Stroke and Bleeding in Atrial Fibrillation Patients. Journal of Stroke and Cerebrovascular Diseases. 2020;29(5):104718.

[57] Zhang T, Tsang W, Wijeysundera H, Ko D. Reporting and representation of ethnic minorities in cardiovascular trials: A systematic review. American Heart Journal. 2013;166(1):52-57.

[58] Felker G, Mann D. Heart Failure E-Book : A Companion to Braunwald's Heart Disease. 3rd ed. Philadelphia PA: Elsevier; 2016.

[59] Blackledge H, Newton J, Squire I. Prognosis for South Asian and white patients newly admitted to hospital with heart failure in the United Kingdom: historical cohort study. BMJ. 2003;327(7414):526-531.

[60] Chaturvedi N. Ethnic Differences in Cardiovascular Disease. Heart. 2003;89(6):681-686.[61] Sun L, Kimmoun A, Takagi K, Liu P, Bader Eddeen A, Mebazaa A. Ethnic differences in acute heart failure outcomes in Ontario. International Journal of Cardiology. 2019;291:177-182.

[62] Choi D, Nemi E, Fernando C, Gupta M, Moe G. Differences in the Clinical Characteristics of Ethnic Minority Groups With Heart Failure Managed in Specialized Heart Failure Clinics. JACC: Heart Failure. 2014;2(4):392-399.

[63] Ziaeian B, Heidenreich P, Xu H, DeVore A, Matsouaka R, Hernandez A et al. Race/Ethnic Differences in Outcomes Among Hospitalized Medicare Patients With Heart Failure and Preserved Ejection Fraction. JACC: Heart Failure. 2017;5(7):483-493.

[64] Kamath S, Drazner M, Wynne J. Characteristics and Outcomes in African American Patients With Decompensated Heart Failure. Archives of Internal Medicine. 2008;168(11):1152. 
[65] Lai E, Grubisic M, Palepu A, Quan H, King K, Khan N. Cardiac medication prescribing and adherence after acute myocardial infarction in Chinese and South Asian Canadian patients. BMC Cardiovascular Disorders. 2011;11(1).

[66] Chong E, Wang H, King-Shier K, Quan H, Rabi D, Khan N. Prescribing patterns and adherence to medication among South-Asian, Chinese and white people with Type 2 diabetes mellitus: a population-based cohort study. Diabetic Medicine. 2014;31(12):1586-1593.

[67] Franciosa J, Ferdinand K, Yancy C. Treatment of Heart Failure in African Americans: A Consensus Statement. Congestive Heart Failure. 2010;16(1):27-38.

[68] Vardeny O, Cavallari L, Claggett B, Desai A, Anand I, Rossignol P et al. Race Influences the Safety and Efficacy of Spironolactone in Severe Heart Failure. Circulation: Heart Failure. 2013;6(5):970-976.

[69] Shekelle PG, Rich MW, Morton SC, Atkinson CS, Tu W, Maglione M, et al. Efficacy of angiotensinconverting enzyme inhibitors and beta-blockers in the management of left ventricular systolic dysfunction according to race, gender, and diabetic status: a meta-analysis of major clinical trials. J Am Coll Cardiol. 2003;41(9):1529-38.

[70] Ponikowski P, Voors A, Anker S, Bueno H, Cleland J, Coats A et al. 2016 ESC Guidelines for the diagnosis and treatment of acute and chronic heart failure. European Heart Journal. 2016;37(27):2129-2200.

[71] Sahni S, Horwich T, Fonarow G. Racial and Ethnic Differences in Heart Failure Etiology, Prognosis, and Management. Current Cardiovascular Risk Reports. 2014;9(1).

[72] Horne R, Weinman J. Patients' beliefs about prescribed medicines and their role in adherence to treatment in chronic physical illness. J Psychosom Res. 1999;47(6):555-67. doi: 10.1016/S0022-3999(99)00057-4.

[73] Stampfer MJ, Hu FB, Manson JE, Rimm EB, Willett WC. Primary prevention of coronary heart disease in women through diet and lifestyle. N Engl J Med. 2000;343(1):16-22.

[74] Bhatnagar A. Environmental determinants of cardiovascular disease. Circ Res. 2017;121(2):162-80.

[75] Egede LE. Race, ethnicity, culture, and disparities in health care. J Gen Intern Med. 2006;21(6):667-9.

[76] Rosengren A, Smyth A, Rangarajan S, Ramasundarahettige C, Bangdiwala SI, AlHabib KF, et

al. Socioeconomic status and risk of cardiovascular disease in 20 low-income, middle-income, and

high-income countries: the Prospective Urban Rural Epidemiologic (PURE) study. Lancet Glob

Health. 2019;7(6):e748-60.

[77] Dhungana RR, Thapa P, Devkota S, Banik PC, Gurung Y, Mumu SJ, et al. Prevalence of cardiovascular disease risk factors: A community-based cross-sectional study in a peri-urban community of Kathmandu, Nepal. Indian Heart J. 2018;70:S20-7.

[78] Eriksen A, Tillin T, O'Connor L, Brage S, Hughes A, Mayet J, et al. The impact of health behaviours on incident cardiovascular disease in Europeans and south Asians - A prospective analysis in the UK SABRE study. PLoS One. 2015;10(3):e0117364.

[79] Brewer LC, Cooper LA. Race, Discrimination, and Cardiovascular Disease. AMA J Ethics. 2014;16(6):455-60.

[80] Hill A, Jones D, Woodworth L. Physician-patient race-match reduces patient mortality [Internet]. 2020 [cited 2020 Aug 23]. Available from: https://papers.ssrn.com/abstract=3211276

\section{Hosted file}

Racial Diaprities Tables.docx available at https://authorea.com/users/354805/articles/478238racial-disparities-and-cardiovascular-disease-one-size-fits-all-approach 